Rabaska

Revue d'ethnologie de l'Amérique française

FERLAND, CLAUDE. Cadiens et voyageurs : un parcours

singulier au Pays d'en-Haut. Québec, Les Éditions GID, 2016, 294 p. ISBN 978-2-89634-306-5

\title{
Gratien Allaire
}

Volume 15, 2017

URI : https://id.erudit.org/iderudit/1041145ar

DOI : https://doi.org/10.7202/1041145ar

Aller au sommaire du numéro

Éditeur(s)

Société québécoise d'ethnologie

ISSN

1703-7433 (imprimé)

1916-7350 (numérique)

Découvrir la revue

Citer ce compte rendu

Allaire, G. (2017). Compte rendu de [FERLAND, Claude. Cadiens et voyageurs : un parcours singulier au Pays d'en-Haut. Québec, Les Éditions GID, 2016, 294 p.

ISBN 978-2-89634-306-5]. Rabaska, 15, 247-250.

https://doi.org/10.7202/1041145ar

Ce document est protégé par la loi sur le droit d'auteur. L'utilisation des services d'Érudit (y compris la reproduction) est assujettie à sa politique d'utilisation que vous pouvez consulter en ligne.

https://apropos.erudit.org/fr/usagers/politique-dutilisation/
Cet article est diffusé et préservé par Érudit.

Érudit est un consortium interuniversitaire sans but lucratif composé de l’Université de Montréal, l’Université Laval et l’Université du Québec à Montréal. Il a pour mission la promotion et la valorisation de la recherche. https://www.erudit.org/fr/ 
marques du travail. Le monument une fois érigé, les ouvriers démantèlent les échafaudages.

Si le « conte est un art ancestral qui consiste à parler dans un micro » comme il l'écrit dans Trad, petit lexique bête et méchant à l'usage des néophytes (chez Planète rebelle, 2013, entrée $\mathrm{n}^{\circ} 12$ ), Faubert transcende cette définition taquine. L'entendre, c'est comme l'avoir devant soi. Un bon conteur est avant tout présence de la voix. Les oreilles ont des yeux pour voir ce qu'elles entendent, et ce qu'elles voient, c'est une parole en marche dans le chemin de la narration. Michel Faubert est un marcheur de contes dans le sentier des mots.

Bertrand Bergeron

Saint-Bruno en Lac-Saint-Jean

Ferland, Claude. Cadiens et voyageurs : un parcours singulier au Pays d'enHaut. Québec, Les Éditions GID, 2016, 294 p. ISBN 978-2-89634-306-5.

C'est un projet ambitieux que Claude Ferland a entrepris il y a plusieurs années et dont il livre les résultats dans cet ouvrage : retracer les Acadiens et Acadiens de souche, voyageurs, qui ont participé à la traite des fourrures, de la période des marchands et des officiers français (début du XVIII ${ }^{\mathrm{e}}$ siècle) à celle des Métis du Nord-Ouest (fin du siècle suivant), en passant par celles de la compagnie du Nord-Ouest et de la Compagnie de la baie d'Hudson. Pour ce faire, l'auteur a puisé dans une vaste documentation : sources manuscrites, sources imprimées, répertoires, banques de données, sites web, etc. Il a utilisé des actes notariés, des récits de voyage, des actes de baptême, de mariage et de sépulture, des biographies, des histoires de paroisses, de nombreuses monographies, des articles de périodiques, etc. Un « travail de moine », pour utiliser une expression consacrée.

Ferland a ainsi pu retrouver des renseignements sur plusieurs voyageurs acadiens ou de souche acadienne, même si leur nombre est très peu élevé, si l'on utilise comme mesure la centaine de contrats d'engagement sur un total de 35 900. Ces hommes étaient originaires de «deux secteurs géographiques en particulier : les paroisses des petites "cadies" » du pourtour du lac Saint-Pierre, et celles au nord du village de Saint-Pierre-du-Portage (L'Assomption)» (p. 57).

Pour illustrer le défi de cette recherche, il suffit de mentionner que l'ouvrage présente plusieurs familles de Landry, comme les Penotte et les Fraule, que les Landry occupent la majeure partie de l'Annexe 1 et que pas moins de douze Joseph Landry y sont inventoriés (p. 221-236), que sept d'entre eux ont été voyageurs et que, pour les distinguer, l'auteur les a 
surnommés Le Réfugié, L’Exilé, Le Maskoutin, Le Yamachichois, Le Sorelois, Le Colvillien, L'Athabascan. Autre exemple de la complexité du travail, la liste des voyageurs appelés « Cadiens » dans les postes de la région Athabascafleuve Mackenzie compte sept noms, avec des surnoms comme Gros Nez, Blanc, Sapin et sont à peu près impossibles à identifier avec précision, sauf peut-être les membres de la famille Lanoue (p. 127-130).

Pour Ferland, ces voyageurs de souche acadienne sont « Les meilleurs des meilleurs » (chap. 4), « Des hommes de valeur » (chap. 7). Selon les contrats d'engagement retrouvés, ils ont exercé plus souvent qu'à leur tour les importantes fonctions de gouvernail et de devant de canot et, même, de guide et d'interprète (p. 196), ce que l'auteur attribue à leur connaissance de la navigation, en mer d'Acadie pour la première génération et parmi les îles de Sorel et le pourtour du lac Saint-Pierre pour les suivantes. Pour appuyer ses affirmations, Ferland a aussi recours aux récits de voyage connus, reprenant, entre autres, ceux d'Alexander Mackenzie vers l'Arctique et vers le Pacifique pour souligner que Joseph Landry, gouvernail, et Charles Doucet ont participé aux deux voyages (p. 88-111). Il y a plusieurs autres documents, repris pour la même raison : les journaux des commis de poste James McKenzie, James Porter, George Keith et Ferdinand Wentzel. Ce sont ces documents, ajoutés aux monographies spécialisées disponibles, qui permettent à l'auteur de présenter une image juste de la vie des voyageurs, de souche acadienne ou autres : conditions des voyages, vêtements, métiers, «tendres liens ». Ils lui ont servi surtout pour suivre de plus près et sur plusieurs générations deux familles formant le « clan Landry-Doucet du nord du lac Saint-Pierre $[, \ldots]$ le seul groupe [acadien] d'importance à pratiquer le métier de voyageur dans cette zone géographique » (p. 59). À travers toute cette documentation, Ferland constate à son tour « l'existence de réseaux sociaux de voyageurs et de traiteurs » (p. 25).

L'ouvrage se termine sur un chapitre intitulé « Cadiens et Métis » pour illustrer que plusieurs « Cadiens » étudiés ont fait comme beaucoup d'autres voyageurs : ils sont restés dans le Nord-Ouest, ont épousé des Amérindiennes ou des Métisses, ont adopté les us et coutumes des postes et ont eu une descendance métisse. Ce que l'auteur illustre ainsi : «De Cadiens, les Lanoue [une famille de Cadiens] sont devenus Métis » (p. 209).

Si l'auteur trace une image relativement juste du groupe choisi à travers les journaux de poste et des voyages utilisés, il faut tout de même souligner qu'elle est fortement marquée par l'expérience de la région de l'Athabasca et du fleuve Mackenzie, une région difficile. Toutefois, la présentation du contexte historique du commerce des fourrures n'est pas aussi solide. Ce n'est pas l'objet central de l'ouvrage, mais elle tend à en diminuer la por- 
tée et la valeur. Les allers-retours chronologiques permettent de suivre les hommes choisis, mais font souvent sauter d'une période à l'autre, dans des contextes différents. C'est le cas surtout à l'époque de la Conquête et de la Déportation (p. 47-50).

L'auteur consacre une partie de l'Annexe 3 à Pierre Gaultier de La Vérendrye. Il y fait une description du rôle des officiers de poste, des marchands et des marchands-équipeurs qui est imprécise. Même s'ils détenaient le droit de commerce à titre de commandant de poste, les officiers, comme La Vérendrye, ne participaient pas directement au commerce des fourrures : ils formaient plutôt une société commerciale avec des voyageurs ou des marchands-voyageurs et les rôles de chacun étaient clairement définis par contrat devant notaire. Le commandant fournissait son droit de commerce et le voyageur ou le marchand-voyageur son expérience du commerce ; le contrat stipulait aussi le partage des bénéfices ; il arrivait de plus en plus souvent que l'officier affermait le commerce du poste, pour s'assurer d'un revenu. Les marchands-équipeurs faisaient rarement partie de ces sociétés ; comme ils fournissaient les marchandises et les avances de fonds requises, ils recevaient les fourrures, mais ne participaient pas au risque des voyages eux-mêmes ${ }^{6}$. Cette forme d'organisation a d'ailleurs été reprise et perfectionnée par les marchands écossais de la Compagnie du Nord-Ouest et de la Compagnie XY.

L'idée de reproduire une grande partie du récit que Joseph Landry de la Rivière-Rouge a fait à l'abbé Jean-Marie Jolys est excellente. C'est un récit exceptionnel puisque la perspective des voyageurs eux-mêmes est peu connue. Cependant, la mise en contexte n'est pas juste : dans sa phrase d'introduction, l'auteur laisse entendre que Landry était au service de la Compagnie du Nord-Ouest lorsqu'il a quitté Terrebonne en juillet 1815 ; ce n'est que par la suite que le lecteur comprend qu'il était alors employé par la Compagnie de la baie d'Hudson (p. 187-188). Landry faisait partie, avec John Clarke et François Decoigne, du premier groupe de voyageurs embauchés en 1815 par Colin Robertson pour faire appliquer par la Compagnie de la baie d'Hudson les tactiques commerciales de leur employeur précédent, la Compagnie du Nord-Ouest. L'auteur mentionne d'ailleurs cette opération ailleurs.

Le lecteur pourrait se passer d'affirmations stéréotypées comme « Les Cadiens, y compris les Métis parmi eux, qui sont généralement reconnus pour leur propension séculaire à la musique et à la danse » (p. 140) ou encore « des Amérindiens, déjà portés à l'ivresse (par la faute des Blancs, admettons-le » (p. 141).

Dans son Cadiens et voyageurs, Ferland présente de solides résultats pour ce qui est de sa recherche sur les voyageurs acadiens ou de souche aca-

6. Voir Gratien Allaire, «Officiers et marchands : les sociétés de commerce des fourrures, 1715-1760 », Revue d'histoire de l'Amérique française, vol. 40, nº 3 (hiver 1987), p. 409-428. 
dienne et accomplit la difficile tâche de reconstituer leur parcours pendant plusieurs générations, de l'Acadie au Nord-Ouest, du Cadien au Métis. Il utilise avec beaucoup de prudence les récits de voyage et les journaux de poste, pour en extraire de précieux renseignements sur les hommes étudiés. Il faut toutefois être plus critique pour ce qui est du contexte commercial de la traite des fourrures.

Gratien Allaire

Professeur émérite, Université Laurentienne

Gagnon, Serge. Familles et presbytères. Québec, Presses de 1'Université Laval, 2013, 174 p. ISBN 978-2-7637-9968-1.

L'historien Serge Gagnon conclut avec Familles et presbytères (2013) sa trilogie sur l'histoire des curés au Bas-Canada. Dans cet essai qu'il qualifie de « biographie collective », Gagnon étudie la période allant de 1790 à 1830 et s'appuie principalement sur la correspondance des curés avec leur évêque. Comme dans Quand le Québec manquait de prêtre (2006) et L'Argent du curé de campagne (2010), l'auteur propose une analyse thématique de cette correspondance. Dans ce dernier ouvrage, Gagnon tâche de déconstruire l'imagerie populaire du curé de campagne habitant un presbytère monumental, une image qui s'appuie davantage sur la réalité des prêtres de la fin du $\mathrm{XIX}^{\mathrm{e}}$ siècle et du début du $\mathrm{XX}^{\mathrm{e}}$ siècle. Il resitue plutôt le service pastoral dans le contexte propre à cette période du développement des paroisses et présente le presbytère comme un espace où s'articulent différents rapports de force. L'ouvrage se structure en trois chapitres. Il aborde d'abord les enjeux reliés à la construction, à l'entretien et à l'usage du presbytère ; le profil socioéconomique des curés de paroisses et ses conséquences ; puis, les liens que les curés entretenaient avec les membres de leurs familles pendant leur service pastoral.

Dans les paroisses du Bas-Canada, l'obligation de fournir une demeure convenable au curé entraîne parfois des situations conflictuelles entre le clergé paroissial, les paroissiens et l'évêché. En première partie, Serge Gagnon présente les enjeux matériels concernant la maison presbytérale. Qui est responsable de sa construction? Qui choisit son emplacement ? Qui doit financer et exécuter les travaux de rénovation? Quels matériaux doivent être utilisés ? Quels sont les droits du curé et les devoirs des paroissiens ? Comment étaient sanctionnés les paroissiens qui tardaient à participer aux travaux ? Dans le contexte de cette époque qui se caractérise par la création de nombreuses nouvelles paroisses, d'un nombre insuffisant de curés pour 